\title{
Sarcoidosis y amiloidosis secundaria, una rara asociación
}

Sarcoidosis and secondary Amyloidosis, an uncommon association

\section{Sebastián Herrera Uribe ${ }^{1 \otimes C \text { CvLAC }}$, Mariana Montoya Castillo², Juan David Berlinghieri-Pérez², Juan Simon Rico-Mesa ${ }^{3}$}

Fecha correspondencia:

Recibido: abril 16 de 2017.

Revisado: noviembre 20 de 2017.

Aceptado: noviembre 24 de 2017.

Forma de citar:

Herrera-Uribe S, MontoyaCastillo M, Berlinghieri-Perez JD, Rico-Mesa JS. Sarcoidosis y amiloidosis secundaria, una rara asociación. Rev CES Med 2018; 32(1): 61-66.

Open access
$\frac{\text { C Derecho de autor }}{\text { Licencia creative commons }}$
$\frac{\text { Ética de publicaciones }}{\text { Revisión por pares }}$
$\frac{\text { Gestión por Open Journal System }}{\text { DOI: http://dx.doi.org/10.21615/ }}$
$\frac{\text { cesmedicina.32.1.7 }}{\text { ISSN 0120-8705 }}$
e-ISSN 2215-9177

Sobre los autores:

1. Médico internista y reumatólogo, Hospital General de Medellín "Luz Castro de Gutiérrez".

Comparte

\section{Resumen}

La sarcoidosis es una enfermedad granulomatosa crónica relacionada frecuentemente con antígenos ambientales e infecciones. Sin embargo, no se ha logrado identificar una causa clara en todos los escenarios. Por su parte, la amiloidosis secundaria se caracteriza por el depósito de proteína amiloide AA en los diferentes tejidos, la cual se asocia a procesos inflamatorios crónicos. Es supremamente infrecuente coincidir con estas dos enfermedades ya que no existe una relación de causalidad directa. A continuación presentamos un caso de un paciente con hallazgos de esta rara asociación.

Palabras clave: Sarcoidosis; Amiloidosis AA; Hipercalciuria.

\begin{abstract}
Sarcoidosis is a chronic granulomatous disease, frequently attributed to environmental antigens (organic and inorganic) and infections. However, it is quite common not to find a clear cause behind this pathology. Alternatively, secondary amyloidosis is characterized my Amyloid AA protein deposition in different tissues, which is associated with chronic inflammation. Nonetheless, it is extremely uncommon to find both sarcoidosis and secondary AA amyloidosis simultaneously provided that there is no a clear causality relationship between both. We present an interesting case of a patient with this uncommon duality.
\end{abstract}

Keywords: Sarcoidosis; Amyloidosis AA; Hypercalciuria.

\section{Introducción}

La sarcoidosis es una enfermedad granulomatosa crónica caracterizada por una respuesta inflamatoria sostenida e inapropiada de tipo celular, que conlleva a la formación de granulomas no caseosos en diferentes tejidos del cuerpo, siendo los pulmones, ganglios linfáticos, piel y ojos, los órganos más afectados. La génesis de esta reacción inflamatoria anómala se ha vinculado a la exposición de una amplia variedad de antígenos inorgánicos, orgánicos, e infecciosos, en individuos genéticamente susceptibles $(\underline{1}, \underline{2})$.

Por su parte, la amiloidosis es un conjunto de desórdenes cuyo sello distintivo es la producción de proteínas erróneamente plegadas y por tanto 
Enero - Abril 2018 - Pág 62

\section{Médico general, Universidad CES.}

3. Visiting Research Fellow in Echocardiography, Department of Cardiovascular Diseases, Division of Echocardiography, Mayo Clinic College of Medicine, Rochester, MN.
Si bien la sarcoidosis no es el desorden inflamatorio más frecuentemente asociado al desarrollo concomitante de amiloidosis se ha descrito una asociación entre ambas. disfuncionales, que tienden a depositarse en diferentes tejidos; estas proteínas se conocen con el nombre genérico de amiloides ( $\underline{3}$ ) La amiloidosis reactiva sistémica es un tipo de amiloidosis que se origina en asociación a diferentes enfermedades inflamatorias crónicas. En ella, el hígado sintetiza amiloide sérico tipo A que tiende a depositarse en el mismo órgano encargado de su elaboración, en el bazo y los riñones (4). Si bien la sarcoidosis no es el desorden inflamatorio más frecuentemente asociado al desarrollo concomitante de amiloidosis se ha descrito una asociación entre ambas (므).

Entre los años 1950 y 1953 se realizaron 52371 autopsias en el hospital universitario de la Universidad de California en Los Ángeles (UCLA), se registraron 503 casos de amiloidosis y 116 casos de sarcoidosis; en siete de las autopsias se encontraron de forma concomitante ambas entidades (므. . A continuación, presentamos el caso de un paciente en el que documentamos ambas enfermedades de forma concomitante.

\section{Reporte de caso}

Paciente masculino de raza negra y 42 años, con cuadro clínico de dos meses de evolución consistente en mialgias generalizadas asociadas a dolor abdominal y síntomas urinarios irritativos. Su historia médica es llamativa por el antecedente personal de tuberculosis pulmonar un año atrás, la cual fue tratada durante seis meses con isoniazida, rifampicina, pirazinamida y etambutol, con adecuada adherencia y respuesta al tratamiento.

Al examen físico se encontró hepato-esplenomegalia y adenopatías bilaterales en cadenas cervicales, axilares e inguinales. En los exámenes paraclínicos se encontró una función renal alterada con tasa de filtración glomerular disminuida (33 mL/ $\mathrm{min} / 1,73 \mathrm{~m}^{2}$ ) asociado a hipercalcemia (calcio sérico $12,1 \mathrm{mg} / \mathrm{dL}$ ), hipercalciuria, nefrolitiasis bilateral, ureterolitiasis derecha, valores disminuidos de hormona para tiroidea y proteinuria en rango nefrótico (cuadro1).

Cuadro 1. Resultados de los exámenes paraclínicos

\begin{tabular}{lll}
\hline Paraclínicos & Resultado & Valores de referencia \\
\hline Creatinina & $2,3 \mathrm{mg} / \mathrm{dL}$ & $0,66-1,25 \mathrm{mg} / \mathrm{dL}$ \\
\hline Calcio sérico & $12,1 \mathrm{mg} / \mathrm{dL}$ & $8,4-10,2 \mathrm{mg} / \mathrm{dL}$ \\
\hline Fósforo & $3,55 \mathrm{mg} / \mathrm{dL}$ & $2,74,5 \mathrm{mg} / \mathrm{dL}$ \\
\hline Hormona paratiroidea & $6 \mathrm{pg} / \mathrm{mL}$ & $7,5-53,5 \mathrm{pg} / \mathrm{mL}$ \\
\hline Proteínas en 24 horas & $5,13 \mathrm{~g} / 24$ horas & $0,04-0,23 \mathrm{~g} / 24$ horas \\
\hline $\begin{array}{l}\text { Anticuerpos para virus de la } \\
\text { inmunodeficiencia humana }\end{array}$ & 0,14 unidades lumínicas & $<0,9$ unidades lumínicas No reactivo \\
\hline $\begin{array}{l}\text { Antígeno de superficie para } \\
\text { virus de la hepatitis B }\end{array}$ & 0,1 & $<0,9$ No reactivo \\
\hline Anticuerpo de la hepatitis C & $0,18 \mathrm{unidades} \mathrm{lumínicas}$ & $<0,9$ unidades lumínicas No reactivo \\
\hline $\begin{array}{l}\text { Prueba rápida de reagina } \\
\text { Vitamina D 1,25 dihidroxi }\end{array}$ & No reactiva & No reactiva \\
\hline
\end{tabular}

Debido a los anteriores resultados se buscó descartar, en primera medida, un síndrome linfo-proliferativo, por lo cual se solicitó biopsia de ganglio inguinal que reveló una arquitectura alterada por la presencia de gran cantidad de nódulos de aspecto granulomatoso, sin necrosis de caseificación y constituidos básicamente por abundantes células 
epitelioides y algunas células gigantes multinucleadas con escasos linfocitos alrededor (fotos 1 y 2 ).

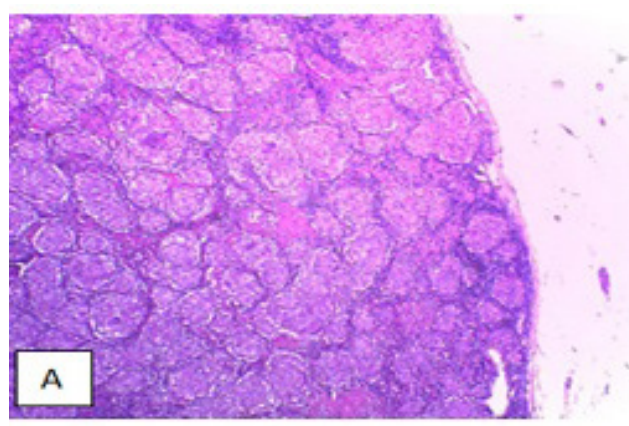

Foto 1. Múltiples granulomas epitelioides en ganglio inguinal

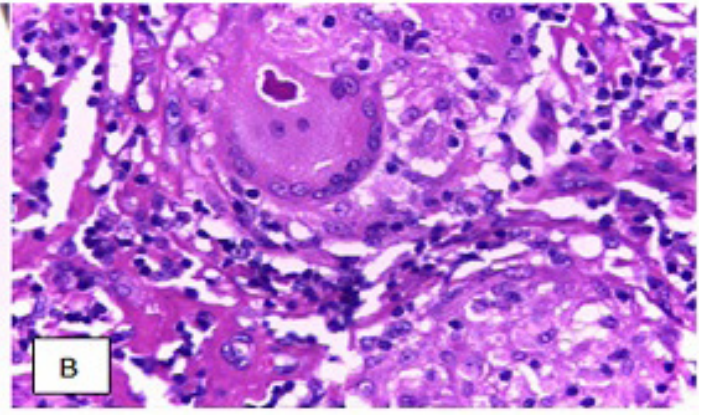

Foto 2. Célula gigante multinucleada de Langerhans encontrada en el tejido de ganglio inguinal

La inmunohistoquímica del ganglio y los estudios de extensión de médula ósea descartaron enfermedad linfoproliferativa. Posterior a los hallazgos en la biopsia y por la presencia de hipercalcemia asociada a hipercalciuria, se sospechó sarcoidosis. Sin embargo, por el antecedente tuberculoso del paciente se solicitó también radiografía de tórax, en la cual se encontraron opacidades bilaterales, aunque sin derrame pleural.

Se decidió entonces complementar el enfoque clínico con una tomografía de tórax de alta resolución en la que se identificaron adenopatías mediastinales peri-bronquiales, algunas calcificadas, con patrón micro y macronodulillar difuso, así como algunas áreas de fibro-

Debido a la presencia de alteración renal se sospechó compromiso renal de la sarcoidosis vs amiloidosis secundaria. Se tomó entonces biopsia de grasa parda abdominal y se realizó tinciones con rojo Congo y hematoxilina - eosina, que evidenciaron material amiloideo, configurando así el diagnóstico definitivo de sarcoidosis y amiloidosis reactivas. sis. Se realizaron fibrobroncoscopia con tinciones para bacilos ácido alcohol resistentes, reacción en cadena de la polimerasa para Mycobacterium tuberculosis por técnica de GeneXpert, y cultivos para bacterias, hongos y micobacterias, los cuales fueron negativos.

Antes de confirmar la sospecha clínica de sarcoidosis se decidió tomar biopsia de las adenopatías peri-bronquiales, la que reportó múltiples nódulos a nivel pulmonar de diferente tamaño, algunos alcanzaron hasta $3 \mathrm{~mm}$, con gran cantidad de células epiteliares y células gigantes multinucleadas, sin linfocitos ni necrosis de caseificación (foto 3 ).

Las tinciones de plata metenamina, ácido peryódico de Schiff y Ziehl-Neelsen fueron negativas para la identificación de hongos y bacilos ácido alcohol resistentes. Así mismo, los cultivos para bacterias, hongos y micobacterias fueron negativos, por lo que se consideró finalmente el diagnóstico de sarcoidosis sin evidencia de malignidad o infección granulomatosa activa al momento.

Debido a la presencia de alteración renal se sospechó compromiso renal de la sarcoidosis vs amiloidosis secundaria. Se tomó entonces biopsia de grasa parda abdominal y se realizó tinciones con rojo Congo y hematoxilina - eosina, que evidenciaron material amiloideo (foto 4), configurando así el diagnóstico definitivo de sarcoidosis y amiloidosis reactivas, por lo que se decidió iniciar manejo con cloroquina, presentando adecuada evolución, mejoría de sus síntomas y de la hipercalcemia. 


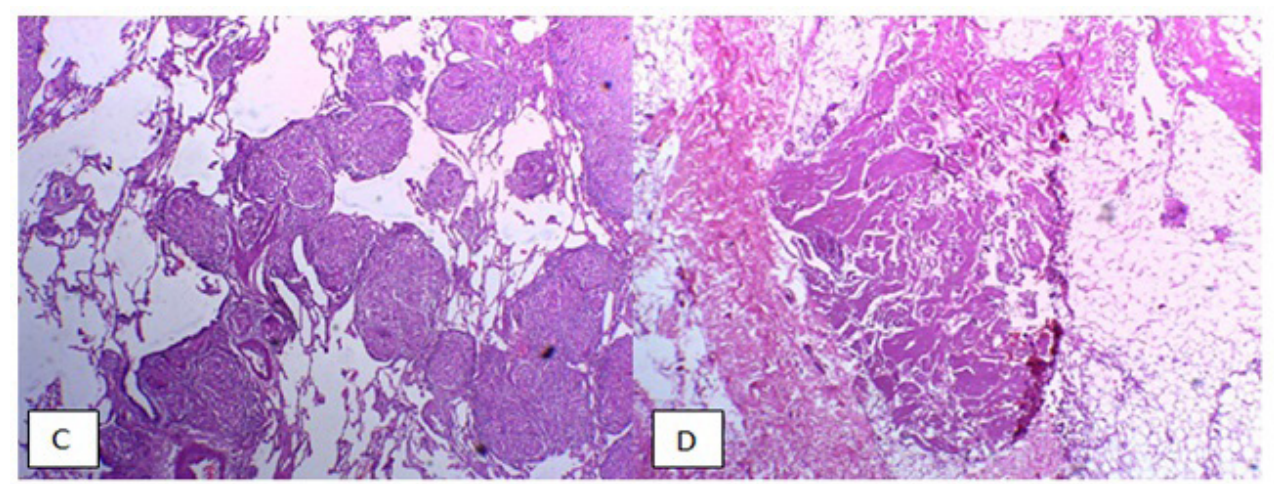

Foto 3. Tejido pulmonar demostrando gran número de células epitelioides
Foto 4. Tinción hematoxilina eosina de grasa abdominal, observándose depósito amiloideo

\section{Discusión}

La sarcoidosis es una enfermedad de difícil diagnóstico debido a la confusión con otras enfermedades con características clínicas similares. La ausencia de una prueba diagnóstica específica y las diferentes condiciones que pueden semejar hallazgos histopatológicos (p.e. infecciones por micobacterias, hongos, malignidad o beriliosis) favorecen la dificultad e incertidumbre en el diagnóstico (ㅇ).

La distribución de la enfermedad es mundial y su incidencia varía de acuerdo a la raza y el sexo. En Estados Unidos de América, la tasa de incidencia anual ajustada a la edad en las personas caucásicas es de 10,9 por 100000 habitantes y en los afro-americanos es de 35,5 por 100 000. En Europa oriental es de 3,7 en 100000 y

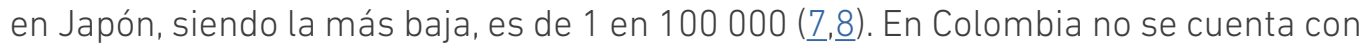
estudios cerca de su prevalencia e incidencia.

La etiología de la sarcoidosis no es por completo entendida, pero se ha propuesto que por el tipo de órganos que se compromete, ocurre como respuesta a antígenos ambientales e inorgánicos) e infecciosos.
La etiología de la enfermedad no es por completo entendida, pero se ha propuesto que por el tipo de órganos que compromete, siendo los pulmones y ganglios linfáticos regionales afectados en un $90 \%$ de los casos, la piel en un $30 \%$ y los ojos en un $25 \%$ (9), el trastorno ocurre como respuesta a antígenos ambientales (orgánicos e inorgánicos) e infecciosos (10). En el paciente descrito se identificó antecedente de tuberculosis pulmonar con tratamiento previo, aunque fueron negativos los estudios para Mycobacterium tuberculosis. Es importante descartar esta enfermedad ya que se ha descrito una asociación importante entre la exposición a antígenos de micobacterias residuales como potenciales causantes de sarcoidosis $(\underline{11}, \underline{12})$.

En cuanto a los trastornos asociados a la sarcoidosis se destaca la presencia de hipercalcemia e hipercalciuria (13). Se estima que $10 \%$ de los pacientes con diagnóstico de sarcoidosis pueden desarrollar hipercalcemia y 30 \% hipercalciuria 14. Las principales causas están ligadas a la conversión de 25-hidroxi-vitamina-D en 1-25 hidroxi-vitamina-D por la expresión de alfa-1-hidroxilasa en los granulomas (15). Se ha propuesto también la presencia de un péptido similar a la paratohormona originado de igual manera en los granulomas (15).

En la descripción del caso se destaca el hallazgo de amiloidosis asociado a sarcoidosis. Esta enfermedad se caracteriza por el depósito de proteínas erróneamente plegadas en el espacio extracelular como fibras insolubles que interfieren con la función normal del tejido (16). Específicamente, la amiloidosis AA es la variedad amiloide más frecuentemente relacionada con afectación del parénquima renal (3). 
La asociación de amiloidosis y sarcoidosis ha sido reportada y se destaca la proteinuria entre los hallazgos más usuales.
Su asociación con sarcoidosis ha sido reportada y se destaca la proteinuria entre los hallazgos más usuales (17). En el paciente descrito llamaba la atención la proteinuria con sedimento blando, lo que hizo sospechar el compromiso. La biopsia de grasa subcutánea abdominal se escogió por un mejor rendimiento y por la necesidad de una buena sensibilidad para evitar procedimientos adicionales (3). Consideramos que el compromiso renal era secundario a la amiloidosis, aunque no fue posible confirmarlo ya que no se realizó biopsia renal.

La importancia de este caso radica en hacer énfasis en una enfermedad poco frecuente y sus alteraciones asociadas, que por el tipo de compromiso clínico e histopatológico puede llegar a ser confundida con enfermedades de mayor prevalencia como la tuberculosis. Es elemental buscar y descartar complicaciones secundarias como amiloidosis en estos pacientes que presentan sarcoidosis acompañados de signos y síntomas de acumulación de proteínas amiloideas.

\section{Aspectos éticos y conflictos de intereses}

El reporte de caso presentado es acerca de un paciente del Hospital General de Medellín. El paciente fue analizado de forma retrospectiva, sin revelar información personal que lo identifique, por lo cual no se utilizó un consentimiento informado. Ninguno de los autores presenta conflicto de intereses personales o económicos ante la preparación de dicho caso clínico.

\section{Bibliografía}

1. Iannuzzi MC. Sarcoidosis. N Engl J Med. 2008 Mar 27;358(13):1402-5.

2. Hamzeh N. Sarcoidosis. Med Clin North Am. 2011 Nov;95(6):1223-34.

3. Merlini G, Seldin DC, Gertz MA. Amyloidosis: Pathogenesis and new therapeutic options. J Clin Oncol. 2011;29(14):1924-33.

4. Falk. Rodney H, Comenzo. Raymond L SM. The systemic amyloidoses. N Engl J Med. 1997;337(13):898-909.

5. James DG, Sharma OP. Overlap syndromes with sarcoidosis. Postgrad Med J 1985;61(719):769-71.

6. Sanjay Mukhopadhyay, Anthony A. Granulomatous Lung disease: an approach to the differential diagnosis. Archives of Pathology \& Laboratory Medicine. 2010 May;134(5):667-90.

7. ATS Boards of Directors and ERS Excetuvie Committee. Statement on Sarcoidosis Am J Respir Crit Care Med. 1999 Aug;160(2):736-55.

8. Morimoto T, Azuma A, Abe S, Usuki J, Kudoh S, Sugisaki K, et al. Epidemiology of sarcoidosis in Japan. Eur Respir J. 2008 Feb 1;31(2):372-9.

9. Iannuzzi M FJ. Sarcoidosis clinical presentation, immunopathogenesis, and therapeutics. JAMA. 2011;305(4):391-9.

10. Iannuzzi MC, Rybicki BA, Teirstein AS. Sarcoidosis. N Engl J Med. 2007:357(21):2153-65. 
11. Mortaz E, Adcock IM, Barnes PJ. Sarcoidosis: Role of non-tuberculosis mycobacteria and Mycobacterium tuberculosis. Int J Mycobacteriology. 2014 Dec;3(4):225-9.

12. Sanz-Martín N, Samillán-Sosa K del R, De Miguel J, Martínez-Miguel P. Renal amyloidosis in leprosy, an infrequent cause of nephrotic syndrome in Europe. BMJ Case Rep. 2016 Aug 3;bcr2016216038.

13. Hilderson I, Van Laecke S, Wauters A, Donck J. Treatment of renal sarcoidosis: Is there a guideline? Overview of the different treatment options. Nephrol Dial Transplant. 2014;29(10):1841-7.

14. Hamzeh N. Sarcoidosis. Med Clin North Am. 2011;95:1223-34.

15. Conron M, Young C, Beynon HL. Calcium metabolism in sarcoidosis and its clinical implications. Rheumatol Oxf Engl. 2000;39(7):707-13.

16. Govender P, Berman JS. The Diagnosis of Sarcoidosis. Clin Chest Med. 2015;36(4):585-602.

17. Rainfray M, Meyrier A, Valeyre D, Tazi A, Battesti J. Book notices. 1988;422-3. 